\title{
Methods to Be Developed for Some First Applications of Mitochondrial Filamentation
}

\author{
Mario Gosalvez \\ Laboratorio y Servicio de Bioquímica Experimental Clinica and Hospital Universitario Puerta de Hierro, \\ Madrid and Majadahonda (1970-2010), Comunidad de Madrid, Spain \\ Email: dmg.secre@gmail.com
}

Received December 25, 2012; revised January 27, 2013; accepted February 7, 2013

\begin{abstract}
A concise review of mitochondrial filamentation and its first possible applications for cancer, psychosis and obesity is provided. Only they are indicated the more essential references mentioning the first possible methods to be developed. Mitochondrial filamentation is a new field based in the more physiological isolation of mitochondria by decreasing the mechanical, thermal and chemical stress. It is from now when this new field could pass to its development assisted by the more capable international capacities.
\end{abstract}

Keywords: Mitochondrial Filamentation; Schizophrenic Psychoses; Obesity

\section{Background}

The new field of mitochondrial filamentation and its first potential applications are just getting underway [1-7], following a series of pilot experiments in our laboratory over the past few decades [8-14]. I should like to give a brief overview of the role that some new methods are playing in this emerging area of physiological bioenergetics, supported by a few references to our contributions as I scarcely have the faculties now to understand other specific contributions. This new field is brimming with promises for medicine, science and society, in general. I cannot and must not be concrete or comprehensive, because I honestly feel that it is now that matters are really beginning to take off, assisted by the most appropriate international capabilities.

Certainly I think that the isolation of all the cellular membranes, not just the mitochondria and chloroplasts, with the lowest possible thermal, mechanical and chemical trauma, would permit the observation of filaments, cones and veils in the majority of life's species. For this endeavor we have already suggested [1], some initial considerations using differential centrifugation. Without doubt, however, by way of example, the filtration and sequential sedimentation would be, among other things, matters for the new technologies to experiment on.

The variety of cellular life is such, and frequently the critical cells are seated in such exquisite locations, that we should strive not to leave any cellular types that we know to be important in any illness unexamined. This would require new and very vital isolation methods for the key cells. To give an important example, we could cite the endothelial cells in the blood vessels of human brains and hearts. Or even in those most identified cells of degenerative neural, muscular, hepatic diseases or in cases of senility [9,11,12,15-18].

\section{Metabolic Control}

The importance of the intimate mechanism of aerobic glycolysis of cancerous cells (2), and perhaps of embryonic cells and primitive cells with an active proliferation, might soon be partially confirmed as a first correlation of the discovery of filamented mitochondria. Nevertheless, we would also now suggest they they ought to study other areas of metabolic control in addition to investigating the systems in which mitochondria intervene. Especially where there is a lot of hydrolysis and synthesis of ATP. Besides this, in cellular systems where there is aerobic glycolysis of more physiological importance, as for example in the retina and the intestinal villi and crypts [3].

The relationship between genome, molecular biology, immunology, anoxia and countless other things with mitochondria has hardly begun. I truly believe that the cellular and multicellular integration of everything with everything will result in the first step towards an advanced science that might allow for the first possible resonances of life with the cosmos to be seen.

Perhaps in the studies of metabolic control a very important step forward would be a less traumatic isolation, not just of mitochondria and all cellular membrane or- 
ganelles, but also different molecules, macromolecules, enzymes, complex multi-enzymes, fibers, cellular associations, tissues and organs, for example, with the greatest physiological integrity possible in respect of their presence in the living.

In biochemistry's infancy it was necessary to proceed by dissecting and re-dissecting everything separately in order to discover the details. We are now getting closer to a second essential process, also quite embryonic: the stage when everything has to be constantly joined and re-joined to everything else so as to clearly distinguish, for example, the different types of cellular lives and deaths [5-7].

\section{Reversal of Cancer}

The continuous recording of personal walking mobility data, the determination of the tumor's weight by new urine analysis throughout the period subsequent from diagnosis to complete cures plus the determination of the leukocyte alteration of the recurring mitochondrial filamentation and defilamentation cycles would, certainly, represent new methods necessary for treatment, diagnosis, nature and prevention of human cancers [4,8-14]. We started some decades ago some techniques for the determination of the respiration and glycolysis in isolated single tumoral cells in an artificial growing medium and the correlation of some mice movements with variations in the vertical force of gravity. We cannot desist from pointing out the importance of those biophysical techniques (8) and of the need to extend their application more widely.

Most certainly, as time passes, better reversal agents and redoubt cytotoxicity than the developments based on our investigations may be found. The isoenzymes of glycolysis enzymes and mitochondria, determined by radioimmuno assay in urine, cooperate markedly with clinical techniques to define a tumor's malignancy. The especially nutritive therapies and concomitant mobility modulation with reversal to normality chronotherapies and with cytotoxicity redoubts will also be important additions for the definitive cure of all cancers. Both for the most well-known hyperplastic types as well as the least studied atrophic ones: especially all the aplastic and hypoplastic neoplasms [8-14]. To be totally transparent with readers I will repeat, this time to the four winds, that in my opinion SIDA is fundamentally a lymphpocytary aplasia with a viral concomitant different to that of aplastic anemia.

\section{Obesity}

I seem to recall that in the 1940's and 50's oxidative phosphorylation un-couplers such as dinitrophenol were tested in the treatment of acute obesity. Naturally the patients lost weight rapidly although their body temperatures increased markedly. These clinical tests were, however, quickly abandoned, as the death rate associated with the treatment was very high.

The most suitable physiological corrections of the alterations of the recurrent filamentation cycle for mitochondrial filamentation-defilamentation which many types of chronic metabolic obesities portrait, and some slim people, could lead in a decade to a focusing, with appropriate efficiency, on these serious problems afflicting society.

The first thing necessary in the diagnosis of patients susceptible to a total cure would most certainly be the application of at least the first three methods combined for metabolic characterization: measuring of personal mobility, diagnosis of the recurrent coupling of mitochondria leukocyte filaments and continuous thermal monitoring.

\section{Schizophrenic Psychoses}

Regardless of the patients' intellectual and emotional capabilities, which in my opinion are a distinguishing feature of a large number of patients with mental disorders, to a degree that the most acute cases are not susceptible to being reintegrated into society, would be as I term it “The Uncontrollable Endorphic Dissension”. I have coined this phrase by coupling the words "Endo and Orphic" so as to be able to talk about the new cerebral glandular transmitters which I postulate can act on innermost uncontrollable dreams. Sometimes they can degenerate into truly horrible tragedies with no apparent cause.

On the positive side, it is not at all unusual to learn about, or to know of, great geniuses in any of science's activities, whether they are frequent depressives, although often they appear jubilant, or more frequently their behavior assumes a cyclical alternating manner. Those often marvelous persons mainly exercise a satisfactory control over their work and family life. Above all over the long term, sometimes decades after their deaths, the History of Science, in capital letters, affords them due recognition.

Nevertheless, in respect of those psychoses referred to above, there are never sufficient traffic wardens for such heavy rush hour traffic in thoughts and actions. More or less peculiar, more or less geniuses, either very depresssive or too excited.

Above all, those afflicted with paranoia in its flowering stage, appear as if they are driving a very high horsepower vehicle with the chassis of a small one. They have no control over it, neither over the accelerator nor the brakes.

In my humble opinion, that disassociation would re- 
flect, in its most essential interior mechanism, an alteration of mitochondrial filamentation-defilamentation cycle of the "Triad epiphyses-pituitary-hypothalamus" with sudden shocks often sharply cosmo-resonating of different "endorphic" molecules and other currently unidentified cerebral glandular transmitters. These would possibly share certain side effects with some already studied endorphins.

Possibly if this were so or somewhat similar, it might be a reason for the evolution of the human species on Earth. This I believe will lead to an unstoppable increase in the number of geniuses produced. Let us not worry ourselves for the moment that destiny is creating ever more incurably insane specimens.

We beseech the relevant scientific communities to contribute non-invasive methods and techniques for the diagnosis of the mitochondrial filamentation located in the brain, to see if our proposition in the future contains a halo of truth.

\section{Preliminary Conclusion}

Evidently the industrial interests that would need to be mustered for these and other applications in this new emerging field do not allow me to be more forthcoming at this stage. But any reader interested in these matters, after carefully reading what is now available, could think about new methods, techniques and ways to assist with the task. If they are able to provide me with some hope and if they are enthusiastic about the desire of trying to cure for all time the sick.

\section{Acknowledgements}

The author is truly indebted to his close friend, Andrew Guy, for his patience and for the submission process to achieve the most possible splendid translation of this text written in Spanish into the very wide English language. Mr. Guy has become already a great scientific translator but not for specific texts.

\section{REFERENCES}

[1] M. Gosalvez, "Mitochondrial Filamentation: Some Methods of Isolation and Assay," IOSR Journal of Pharmacy and Biological Sciences, Vol. 4, No. 4, 2012, pp. 37-39. doi:10.9790/3008-0443739

[2] M. Gosalvez, "Metabolic Control of Respiration and Glycolysis of Tumoral Cells," Advances in Biological Chemistry, Vol. 3, No. 1, 2013, pp. 86-89.
[3] M. Gosalvez, “Cancer as a Therapeutic Agent?” Unpublished.

[4] M. Gosalvez, "Reversal of Cancer by Dual Strategy," Journal of Cancer Therapy, Vol. 4, 2013, pp. 518-520.

[5] M. Gosalvez, "Cancer Reversal and Mitochondrial Filamentation,” Unpublished.

[6] M. Gosalvez, “The Great Planetary Oxygen Cycle?” Unpublished.

[7] M. Gosalvez, “Extension of Metabolic Life?” Unpublished.

[8] M. Gosalvez, “Cancer Reversal by Dual Strategy,” National Center of Medical Research, Madrid, 1992.

[9] M. Gosalvez, "Oxygen Production by Filament Mitochondria. Importance in Cancer and Neurodegeneration,” Anales de la Real Nacional Academia de Medicina, Madrid, Vol. 115, No. 3, 1998, pp. 747-756.

[10] M. Gosalvez, "Reversion of Cancer and Mitochondrial Filamentation,” Anales de la Real Nacional Academia de Medicina, Madrid, Vol. 117, No. 4, 2000, pp. 825-834.

[11] M. Gosalvez, M. Blanco, P. S. Testillano and M. C. Risueño, "Warm Low Stress Isolated Filamented Mitochondria," 2nd World Congress of CMB. Cellular and Molecular Biology, Paris, 1996.

[12] M. Gosalvez and M. Blanco, "Oxygen Production by Filamentous Mitochondria," Proceedings of the 26th Annual Meeting of ISOTT, Budapest, 23-28 August 1998.

[13] M. Gosalvez, “Mitochondrial Filamentation,” Proceeding of the AACR 101st Meeting, Washington DC, April, p. 20.

[14] M. Gosalvez, "Reversal of Cancer by Dual Strategy: A New Molecular Target?” Proceedings of the AACR 103rd Meeting, Chicago, 31 March-4 April 2012, p. 1118.

[15] J. M. Gobernado, M. Gosalvez, C. Cortina, M. Lousa, C. Riva, A. Gimeno, "Mitochondrial Functions in Chronic Muscular Atrophy,” Journal of Neurology, Neurosurgery \& Psychiatry, Vol. 43, No. 6, 1980, pp. 546-549.

[16] F. O’Connor, J. L. Castillo-Olivares, M. G. Eguaras, A. Juffe, M. Gosalvez and D. Figera, “Anoxic Cardiac Arrest: Its Effect on Myocardial Mitochondrial Metabolism,” The Journal of Cardiovascular Surgery, Vol. 16, No. 5, 1975, pp. 493-499.

[17] A. Bootello, E. Fernandez-Cruz, P. Escartin, M. F. Blanco, M. Gosalvez and J. M. S. De Arana, "Injurious Effect on Rat Liver Mitochondria by Lymphocytes from Patients with Primary Biliary Cirrhosis,” Clinical \& Experimental Immunology, Vol. 24, No. 2, 1976, pp. 374-377.

[18] J. Diaz Gil, I. Rossi, P. Escartin, J. M. Segovia and M. Gosalvez, "Mitochondrial Functions and Content of Microsomal and Mitochondrial Cytochromes in Human Cirrhosis," Clinical Science \& Molecular Medicine, Vol. 52, No. 6, 1977, pp. 599-606. 